Discourse in Filipino Philosophy

\title{
Tao bilang Tagpuan: Roque Ferriols, San Agustin, at ang Puntong Omega
}

Mark Joseph T. Calano

\begin{abstract}
In his book Pilosopiya ng Relihiyon, Roque Ferriols argued that the encounter between God and the human person can be concretely experienced in the attempt of being human. What does he mean by this statement? This paper tries to understand this statement in three parts. The first part analyzes Ferriols's assertion of the role of damdamin, the personal nature of God, and our encounter with others and God. In trying to understand this relational (rather than conceptual) encounter with God, we move to Augustine of Hippo's discussion of remembering, knowing, and willing (or loving) as the mental faculties that are reflective and analogical of the Trinity in the human person; the second part situates the discourse within a Christian narrative that argues for Christ as the exemplar and fullness of humanity. The third part discusses Ferriols's treatment of Teilhard de Chardin's Omega Point, where evolution leads us to further creation instead of destruction; this part connects with the second part in that Ferriols (drawing from de Chardin) speaks of the Omega Point as 'possibly' referring to Christ. It further discusses how we are invited into this personal love that unites and creates us in the process of being more human.
\end{abstract}

Keywords: Augustine, Ferriols, human person, Omega Point

(c) 2018 Mark Joseph T. Calano

https://www.kritike.org/journal/issue 22/calano june2018.pdf

ISSN 1908-7330 


\section{Pambungad}

Diyos ang tao ayon sa kaniyang sariling larawan, ayon sa larawan ng Diyos siya nilalang; nilalang niya sila na lalake at babae. At sila'y binasbasan ng Diyos, at sa kanila'y sinabi ng Diyos, "Kayo'y magpalaanakin, at magpakarami, at kalatan ninyo ang lupa, at inyong supilin; at magkaroon kayo ng kapangyarihan sa mga isda sa dagat, at sa mga ibon sa himpapawid, at sa bawa't hayop na gumagalaw sa ibabaw ng lupa" (Genesis 1:26-28). ${ }^{1}$

$\mathrm{K}$

adalasang ginagamit ang sipi sa itaas upang tukuyin ang dibinong pagmemeron at pagkawangis ng tao. Ngunit para kay Roque dibinong nagmemeron: mahal at banal. ${ }^{2}$ Ayon sa kaniya, mahal ang katagang ginagamit natin upang tukuyin ang karanasan ng pagbibigay halaga. Ngunit hindi lamang ito usapan ng halaga, may ginagampanan din ang kilos ng pagibig. Sa ating pagmamahal sa kapuwa ay natatauhan tayo sa isang kilos na maka-Diyos. Paliwanag ni Ferriols, "Pag-ibig ang pinagsisilangan ng pagmamahal, na humihigit pa sa pag-ibig at umiiral sa pinakabuod ng ating pagkatao ... Ang buod ng karanasang mahal ay isang pagsasaatin ng Maykapal at isang pagsasa-Maykapal natin." ${ }^{3}$ Banal naman ang salita na ginagamit upang tukuyin ang isang paggalang sa pakikipagugnay sa Diyos na higit pa sa anumang panlabas na kilos; isang ugnayan sa Maykapal na "nasa kalooban ng tao" at "maaaninagan, mahuhulaan lamang ng kaniyang kapuwa." ${ }^{4}$ Ang banal ay isang pagsamba na kumikilos sa kalooban ng tao ngunit ay sabay na nagpapakita sa kilos na "sumasatao ang Maykapal at sumasa-Maykapal ang tao." ${ }^{5}$ Para kay Ferriols, kahit na magkaiba ang diin at ang tinutukoy ng banal at ng mahal, parehong karanasan naman ang tinutukoy ng dalawang kataga na ito.

Kung titingnan ang kahulugan ng banal at mahal, ang dalawang kataga ay kumikilos sa kalooban ng tao patungo sa pagmamahal at pagkakawanggawa sa kapuwa at sa pagpuri at pagsamba sa Diyos. Habang mayaman ang larangan ng mahal (at banal at may paghiwatig ito sa Diyos),

${ }^{1}$ Lahat ng sipi sa Bibliya sa papel na ito ay binago ng kaunti ng may-akda at mula sa Ang Banal na Kasulatan na Kinalalamnan ng Matanda at Bagong Tipan na Isinalin sa Wikang Tagalog (Manila: Philippine Bible Society, 1982).

2 Roque Ferriols, "Karanasang Mahal-Banal," in Magpakatao: Ilang Babasahing Pilosopiko (Quezon City: Office of Research and Publications, Ateneo de Manila University, 1999), 173-181.

${ }^{3}$ Ibid., 173-174.

${ }^{4}$ Ibid., 174.

${ }^{5}$ Ibid.

(c) 2018 Mark Joseph T. Calano

https://www.kritike.org/journal/issue 22/calano june2018.pdf

ISSN 1908-7330

(c) BY-NC-ND 
nakakagat naman ang ugnayan ng mahal at banal sa ating pagpapakatao. Sa Pilosopiya ng Relihiyon, nilinaw ni Ferriols na ang kadalasang tagpuan ng tao at ng mahal-banal ay makikita sa pagsusumikap ng tao na makipagkapuwa tao. ${ }^{6}$ Ngunit hindi niya ito binigyan ng paliwanag.

Layunin ng papel na ito na ipaliwanag kung papaano nagaganap ang tagpuan ng tao at ng banal sa ating pagsusumikap makipagkapuwa; paguusapin ko sina Roque Ferriols, Agustin ng Hippo, at Teilhard de Chardin upang mabigyang linaw kung papaanong ang tao ay tagpuan ng banal, kung papaanong ang malay-tao ay kawangis ng Banal na Santatlo, at kung papaanong ang buong sannilikha ay uuwi sa puntong Omega. Binubuo ng tatlong bahagi ang papel na ito. Ang unang bahagi ay isang paglalahad o paguulit ng kaisipan ni Ferriols na umiikot sa pag-uunawa sa tao bilang tagpuan ng mahal-banal. Upang maintindihan ito, aking ipaliliwanag ang papel na ginagampanan ng damdamin, ang pagkapersonal ng Banal, at ang tao bilang tao. Ang pangalawang bahagi ay isang pagsubok na gamitin ang kaisipan ni Agustin upang ipaliwanag at sabay itulak ang kahulugan ng talata na tao ang tagpuan sa banal. Dito ko ipinaliwanag ang mga kilos ng malay-tao bilang paggunita, pag-unawa, at paghangad, at ang kanilang ugnayan sa Banal na Santatlo. Ang ikatlong bahagi naman ay isang pagbabalik sa Pambungad sa Metapisika at sa usapin dito ukol sa Puno ng Buhay, Puntong Omega, at Pagibig. ${ }^{7}$

\section{A. Damdamin, Personal, Tao}

Sinimulan ni Ferriols ang kaniyang talakayan ukol sa pakikipagtagpo sa banal sa tungkulin at gampanin ng damdamin; taliwas ito sa nakasanayang kilos ng pilosopiya na nagsisimula sa isip. Habang mahalaga ang papel na ginagampanan ng isip, may kakaibang uri ng paggising na idinudulot ang ating mga damdamin. ${ }^{8}$ Ayon kay Ferriols, ang mga pagbabago na ito rin ang nagsasangkot sa ating kalooban sa isang malalim na uri ng pakikipagkapuwa tao. Binigyan niya ng diin na ang simula ng bawat pakikipagtagpo sa mahal-banal ay hindi nagaganap sa larangan ng isip, bagkus ay nagsisimula at nagaganap sa larangan ng ating mga damdamin. Sa pagkagat ng kaniyang pagtalakay sa aspeto ng damdamin, iniwasan ni Ferriols ang mga tinatanong ng isipan; ito ay sapagkat hindi

${ }^{6}$ Roque Ferriols, Pilosopiya ng Relihiyon (Quezon City: BlueBooks, Ateneo de Manila University Press, 2014), 7.

7 Roque Ferriols, "Theological Aspects of Cultural Adaptation," in Pagdiriwang sa Meron: A Festival of Thought Celebrating Roque J. Ferriols, S.J., ed. by Nemesio S. Que and Agustin Martin G. Rodriguez (Quezon City: Office of Research and Publications, Ateneo de Manila University, 1997), 223-237.

${ }^{8}$ Ferriols, Pilosopiya ng Relihiyon, 1. 


\section{TAO BILANG TAGPUAN}

tinatanong ng damdamin kung meron o kung totoo ba; ${ }^{9}$ may kakaibang uri ng pag-alam na dulot ng damdamin.

Tila baga may kilos ang damdamin na hindi katulad ng ginagawa ng isip; humihiwatig ang kilos ng damdamin. Paliwanag ni Ferriols, "Ang damdamin na hindi lamang nagpapamalay sa kalagayan ng katawan kundi may pinagmumulan at may tinutunguhan, kung kaya't ang tinutunguhan ay nagbibigay-balangkas sa malay-tao ... ang ganyang damdamin ay maaaring pagmasdan, masusing tuonan ng pagsusuri." ${ }^{10}$ Sa pagsisimula sa damdamin, maaaring sabihin na penomenolohiya ang metodo ni Ferriols. Ngunit ayon sa kaniya, ang metodo ng pagmamasid sa balangkas ng malay-tao ay hindi penomenolohikal, ngunit ito ay maka-penomenolohiko. Mahirap bigkasin ang metodo na ito, ngunit maaari naman itong mahiwatigan sa mga susunod na bahagi ng papel.

Kung ang simula ng ating diskurso ay ang isip, maaaring pag-usapan ang Diyos sa isang unibersal na pamamaraan at sa ganyang kalagayan ay masasabi na impersonal ang Diyos. Ngunit sa pagkagat ng ating karanasang banal sa damdamin, tila baga may isang uri ng pagkapersonal ang Diyos. Impersonal o Personal? Habang may mga nagsasabi na ang Diyos ay impersonal (at sa ganyang kalagayan ay higit sa atin at dakila), meron din naman nagsasabi na ang Diyos ay personal (at sa ganyang kalagayan ay binibigyan halaga ang tao). Sa ating damdamin, nakararating tayo sa isang personal na kamalayan ng Diyos na parating buo at kumpleto sa karanasan ng pakikipagkapuwa gamit ang ating kalooban. May mas mahabang paliwanag si Ferriols:

Kapag ang nabiyayaan ng pakikipagtagpong personal sa banal ay umaakyat sa mataas na bundok at natatanaw niya ang malawak na kapatagan sa ibaba na umuunat hanggang sa dagat, at ang dagat naman na umuunat hanggang sa langit, habang ang langit naman ay umaakyat at, mula sa itaas, ay sinusubukan siya ... dumarating lahat ito sa kanya bilang salita sa kanya ng banal o kaya katahimikan ng banal. Palagi siyang nakikinig sa salita o katahimikan ng banal. Kapag siya'y nanliliit, ito'y sapagkat nadarama niya ang paglapit sa kanya ng walang-hanggan na pagkalinga at pagbibigayhalaga. Ang bawat pagharap at pagdanas sa banal ay natatablan ng pakikipagkapuwa sa kanya. Araw-araw na pangyayari ang pakikipagsagutan ng kalooban ng tao

${ }^{9}$ Roque Ferriols, Pambungad sa Metapisika (Quezon City: BlueBooks, Ateneo de Manila University Press, 2014), 213-214.

${ }^{10}$ Ferriols, Pilosopiya ng Relihiyon, 10. 
at ng ubod lalim na kalooban ng banal. Pangyayari na kung minsan kaluwalhatian kung minsan konsumisyon, pero palagi pa ring pangyayari. ${ }^{11}$

Sapagkat may personal na aspeto ang banal, hindi ito maaaring sakupin ng konseptong unibersal. Ang karanasan ng personal na pakikipagtagpo sa banal ay palagiang kakaiba at magkaiba; ito ang bumubuo sa kuwento ng patuloy na pakikipagtagpo sa banal na personal na sabay tumatawag at tumutugon sa tao. ${ }^{12}$

Sapagkat personal ang mahal-banal, tinawag ito ni Ferriols na Diyos, "ang ubod na meron na personal sa kaniyang kaganapan."13 Dito tayo matatauhan na ang pakikipagtagpo sa Diyos ay kailanman higit sa anumang uri ng pagkokonsepto at paglalarawan. ${ }^{14}$ Dito nagiging mahalaga ang kaniyang pagtalakay sa damdamin. Sapagkat ang pagkokonsepto at paglalarawan ay kilos ng isip, mauunawaan natin na mahihiwatigan lamang ang banal at ang paghihiwatig ay bahagi ng kilos ng damdamin. Ngunit dahil pahiwatig lamang ang ating pinakamalapit na mararating sa harap ng Diyos, babalik tayo sa pinaka-ordinaryo at simpleng karanasan ng pakikipagtagpo sa ating kapuwa tao. Sa pagtingin at paglapit sa karanasan ng pakikipagkapuwa, nahihiwatigan natin ang pakikipagtagpo sa banal.

Sa ating pag-unawa sa ating pakikipagtagpo sa ating kapuwa, nagkakaroon tayo ng ideya sa kung ano ang nagaganap sa ating tagpuan: "sumasaiyo sila at sumasakanila ikaw." 15 Sa salitang Latin, ito rin ang kahulugan ng praesens na maaari daw hatiin sa salitang ens na nagmemeron at sa prae na ang kahulugan ay sa harapan. Ang pakikipagtagpo ay maaaring intindihin bilang "[p]akikipagtagpo ng kalooban sa kalooban." 16 Mahalagang intindihin na ang tagpuan ay nasa kalooban ng tao, bahagi ng kaniyang malay-tao, ngunit ay nasa looban. May pagsasa-isa't isa sa kalooban ng bawat kalooban na nakikipagtagpo.

Ang pagpresensiya ay nagaganap sa iba't ibang kilos ng malay-tao: paggunita, pag-unawa, at paghangad; ngunit ay higit pa sa lahat ng kilos na ito ang bukal ng pagprepresensiya. May tunggalian na nagaganap sa pagbuo at sa laro ng presensiya: lapit-layô. Sa paliwanag ni Ferriols,

${ }^{11}$ Ibid., 12.

${ }^{12}$ Ibid., 13.

${ }^{13}$ Ibid.

${ }^{14}$ Ibid. Tingnan din ang Jacklyn Cleofas, "Konsepto at Pag-asa ayon kay Ferriols," in Perspectives in the Arts and Humanities Asia (Special Issue: Philosophy and Roque J. Ferriols, SJ), 5:1 (2015), 21-40.

${ }^{15}$ Ferriols, Pilosopiya ng Relihiyon, 14.

${ }^{16} \mathrm{Ibid}$. 


\section{TAO BILANG TAGPUAN}

Kung mas tumitindi ang pakikipagkapuwa, kung mas naglalapitan, nagtatalaban ang loob, mas natatauhan na ang kapuwa ay may kalaliman na sagrado, may hiwaga na hindi maaring ariin ninoman. Kaya't may nagaganap na magalang na pag-urong, mapitagang paglayô. Ngunit, kung mas gumagalang, kung mas namimitagan ang tao sa kapuwa ay lalo siyang natatauhan na ubod ng magaling at mahalaga ang hiwaga ng kapuwa, kaya't lalo siyang naaakit at lalo siyang napapalapit. ${ }^{17}$

Sa ganitong pag-uunawa, bumubuo tayo ng pamayanan ng mga malay-tao na nakikipagtagpo sa harap ng dinamikong sansinukob. Ngunit nabanggit lamang natin ang kilos ng tao sa kaniyang pakikipagtagpo sa tao. Maari bang sabihin na ganito rin ang kilos ng pakikipagtagpo sa banal?

Tila ang batayan ni Ferriols ay ang kaniyang personal na paguunawa na kadalasan o madalas pa sa kadalasan "ang banal ay lumalapit sa tao sa loob mismo ng pagsisikap ng taong makipagtagpo sa tao."18 Sinuportahan niya ito ng obserbasyon mula sa iba pang mga rehiliyon na nagkakaisa sa pagsisikap na galangin ang kapuwa-"na sa pakikipagkapuwa-tao ay tumutubo ang paniniwala sa banal."19 Ang katapatan na ito sa kapuwa ang siyang nag-uudyok sa atin na buoin ang sarili sa harap ng tentasyon na sirain ito. Ang kapuwa, na maaari ring Diyos, ang siyang "umuudyok, tumatawag, nagbibigay-loob" 20 sa atin. Papaano at saan natatagpuan ang Diyos sa kapuwa at sa tao?

\section{B. Malay-tao kay Agustin}

May nag-iisa at natatanging pagkakataon sa Bibliya na mababasa na ang tao ay tagpuan sa banal; ito ay sinipi na natin sa simula ng papel na ito. Sa siping nabanggit, mababasa sa pagpapahayag ng Diyos kung sino ang tao. Alam natin na ang tao ay may hinihiwatig ukol sa kaniyang kawangis. Ito ang dahilan kung bakit mahalaga ang Exodo 20:4 kapag sinusubok unawain ang Genesis 1:26-28. Sabi nga ni Tomas de Aquino, "hindi natin kayang alamin kung ano ang Diyos, kaya lamang natin alamin kung ano hindi Siya." 21

${ }^{17} \mathrm{Ibid} ., 15$.

${ }^{18} \mathrm{Ibid} ., 16$.

${ }^{19} \mathrm{Ibid}$.

${ }^{20} \mathrm{Ibid}$.

${ }^{21}$ Tomas de Aquino, Summa Theologica [All Complete \& Unabridged 3 Parts, Supplement $\mathcal{E}$ Appendix, and Annotations], translated by Fathers of the English Dominican Province (New York: NY, Benzinger Brother, Inc., 1948), I.I. q.1-8. Akin ang salin. 
Sa konteksto ng kaniyang pagiging Katoliko at Heswita natin makikita kung bakit ang pamimilosopiya ni Ferriols ay isang pagsubok na bigkasin at kilalanin si Kristo. ${ }^{22}$ Para sa kaniya, malinaw ang paanyaya na hubugin ang ating pagkatao na kawangis ni Kristo; iyan din ang pamamagitan ng kaniyang pakikipagtagpo kay Kristo. Dito muling papasok ang damdamin sapagkat maaari lamang nating gayahin at tularan ang mga bagay-bagay kung tunay natin itong iniibig; ang maging kaisa ang tao na hindi natin iniibig ay malapit na sa imposible. Kung ang pakikipagtagpo sa Diyos ay matatamo lamang sa pagsunod kay Kristo (ang wagas na kawangis ng Diyos), dito makikita at mauunawaan kung bakit kinakailangan hubugin ang ating pagkatao ayon sa Diyos Anak. Ang posibilidad nito ay nakakagat sa artikulo ng ating pananampalataya na sapagkat si Kristo ay Anak ng Diyos na naging tao-ang Salita ng Diyos na Nagkatawang Tao-siya ay tao rin. Si Kristo ang natatanging imago Dei, sapagkat si Kristo ang Anak ng Diyos na siya ring sukdulang kawangis ng Ama. Bilang Anak ng Diyos, bilang natatanging Anak ng Diyos, si Kristo ay masasabi rin natin na natatangi at nag-iisang kawangis ng Ama. Sa ganitong pananaw, ang tao ay hindi ang natatanging imahe ng Diyos, at ito ay isinasaad ng Genesis 1:26, "Lalangin natin ang tao sa ating larawan, ayon sa ating wangis." Kung iintindihin natin sa kontekstong ito, nangangahulugan na tayo ay hinulma sa imahe ng Diyos Anak na kawangis naman ng Diyos Ama. Samakatuwid, ang tao ay hindi maaaring maging imago Dei na hiwalay sa ating ugnayan kay Kristo Hesus.

Habang naniniwala si Agustin na nakakagat ang ating pagiging imago Dei kay Kristo, ito ay hindi sapat. Sa kaniyang De Trinitate, ating mauunawaan ang konteksto ng pagtalakay ni Agustin sa kaniyang pilosopikal na pag-unawa sa misteryo ng Banal na Santatlo. Katulad ng paglalahad ni Ferriols, ginamit ni Agustin ang tao upang mas lalong masumpungan ang Diyos. ${ }^{23}$ Sa unang pitong libro ng De Trinitate, nagbigay si Agustin ng mga Biblikal na batayan sa doktrinang ito. ${ }^{24}$ Ngunit matapos ang pitong libro ay tila kulang pa rin ang kaniyang natalakay ukol sa Diyos Ama, Anak, at Espiritu Santo; hindi pa malinaw kung bakit ang Diyos ay may tatlong persona. Kaya sa ikawalong libro, natanto niya na hindi niya

\footnotetext{
${ }^{22}$ Roque J. Ferriols, Sulyap sa Aking Pinanggalingan, ed. by Leovina Ma. Garcia (Quezon City: Ateneo de Manila University Press, 2016).

${ }^{23}$ Habang may tonong teolohikal ang akda, iginigiit ng may-akda na ito ay pilosopikal batay sa argumento ni Marion. Tingnan ang Jean-Luc Marion, "On the Foundation of the Distinction between Theology and Philosophy," ed. by Philippe Capelle-Dumont, trans. by John Carlo P. Uy and Eduardo Jose C. Calasanz, in Budhi: A Journal of Ideas and Culture, 13:1-3 (2009), 15-46. Ayon sa kaniya, hindi pa itinuturing na magkahiwalay na disiplina ni Agustin ang teolohiya at pilosopiya sapagkat ang paghihiwalay na ito ay dulot lamang ng kaisipan at pagkokonsepto ni Tomas de Aquino.

${ }^{24}$ Agustin, On the Trinity, ed. by Gareth B. Matthews, trans. by Stephen McKeena (Cambridge: Cambridge University Press, 2002), Books I-VII.
} 


\section{TAO BILANG TAGPUAN}

maaaring tignan ang Diyos ng harapan, at doon siya nagpasya na hanapin ang Diyos sa kaniyang kawangis. Sapagkat binanggit sa Bibliya na ang tao ay kawangis ng Diyos at tinitiyak ng pananampalataya na ang iisang Diyos ay Santatlo, napagtanto niya na marahil posibleng mahiwatigan ang Banal na Santatlo sa tao. Samakatuwid, ipinahihiwatig niya na sa tao, na nilikhang kawangis ng Diyos, natin masusumpungan ang misteryo ng di-nilikhang Santatlo.

Habang tinatanggap ni Agustin na ang tao ay kawangis ng Anak ng Diyos, hindi naman niya tinatanggap na tayo ay kawangis lamang ng ikalawang persona. Malinaw ang wika ng Bibliya na tayo ay nilikhang kawangis ng Diyos. Kaya raw nakasulat sa Genesis 1:26 na "lalangin natin ang tao sa ating larawan, ayon sa ating wangis" at hindi "lalangin natin ang tayo sa iyong larawan, ayon sa iyong wangis." Sapagkat ang Banal na Santatlo ay magkakapantay-pantay, at iisang Diyos, sumusunod na ang kawangis ng isa ay kawangis ng lahat; hindi lamang tayo kawangis ni Hesus kung hindi ay kawangis din ng Banal na Santatlo.

Sa pagtingin sa tao, nasumpungan ni Agustin kung ano ang Diyos. At sapagkat alam niya sa kaniyang kalooban na ang Diyos ay Ama, Anak, at Espiritu Santo, nahihiwatigan niya ang isang balangkas na sumasalamin sa dibinong misteryo. Para kay Agustin, ang pananalamin ng tao sa misteryo ay sapat na upang maunawaan ang Diyos. Dito makikita ang hangarin ni Agustin na bigyang wangis ang Diyos mula sa kaniyang konkretong pagdanas ng tao at kapuwa. Ibig sabihin, habang Diyos ang sinusubukang bigkasin, ang kaniyang metodo at pamamaraan ay hango pa rin sa kung ano ang kayang abutin ng isip at damdamin.

Ngunit sa isang kakaibang pamamaraan, hindi hinanap ni Agustin ang Diyos sa kaluluwa ng tao; bagkus ay sinayasat niya ang ating malay-tao. Nakita niya ang isang balangkas na bumubuo sa malay-tao bilang paggunita, pag-unawa, at paghangad; itong kilos ng malay-tao rin ang pinakakaganapan ng ating pagiging tao (at ito ay binubuo ng paggunita, pag-unawa, at paghangad sa Diyos). Iba ang kilos ng paggawa sa kakayahan at kilos ng malay-tao. Kung iintindihin ang paggunita, pag-unawa, at paghangad bilang simpleng kakayahan ng malay-tao, maaaring isipin na naroon lamang ito; ngunit kapag uunawain ang tatlo bilang mga kilos ng malay-tao, matatanto natin na ito ay kinakailangang maisakatuparan at magawa.

Ngunit bakit pinili ni Agustin ang tatlong kilos ng malay-tao (paggunita, pag-unawa, at paghangad) upang kumatawan sa Ama, Anak, at Espiritu Santo? Sa totoo lang, hindi agarang naisip ni Agustin na bumaling sa mga kilos ng malay-tao upang kumatawan sa Banal na Santatlo. Ang nais lamang niya ay bigyang liwanag (marahil sa pamamagitan ng analogia) ang eternal na ugnayan ng Anak (ang Salita) at ng Ama sa pamamagitan ng Espiritu Santo. Samakatuwid, nais lamang ni Agustin na makapagbigay ng 
posibleng analogia sa ugnayan ng Banal na Santatlo. Sinimulan niya ito sa pagtalakay ng dalawang saligan ng kilos ng isip: ang pandama at hilig sa mga bagay-bagay. Ngunit alam din niya na ang misteryo ng Santatlo ay ang misteryo ng Diyos sa kaniyang persona; at hindi ang misteryo ng Diyos kaugnay ang kaniyang san-nilikha. Kung ganoon, kinakailangan ni Agustin hanapin sa kaniyang kalooban ang isang analogia upang maipahiwatig ito; at dito, hindi sapat ang kakayahan ng isip na damahin at hiligin ang mga bagay. Kaya, hinanap niya ang kakayahan ng ating malay-tao na pagmunihan ang sariling pag-iisip at pagmamahal. Sa pamamagitan ng pagmumuni-muni sa kaniyang persona, binubuo ng sarili (gamit ang pag-uunawa) ang kaniyang persona; maaari ring sabihin na gamit ang ating malay-tao, bumubuo tayo ng kuru-kuro tungkol sa ating persona. Ngunit papaano? Sa pamamagitan ng pag-uugnay ng mga kuru-kuro gamit ang iba't ibang wika, nabubuo at napagpapatuloy ang kaalaman at karunungan; ang pagdating at ang pagbigkas nito ay maihahambing sa proseso ng pagbuo at pagsilang. ${ }^{25}$ Iyan ang ginamit ni Agustin upang tukuyin ang kaniyang kakayahan na bumuo ng konsepto sa kaniyang pag-unawa; ang katagang mabubuo at maisisilang sa proseso ay tinawag niyang 'salita.' Kung 'salita' ang gamit niya upang pagusapan ang kaniyang kakayahan na umunawa at pagmunihan ang isip, natanto niya na 'Salita' (o sa Griyego ay logos) rin ang gamit sa Ebanghelyo ni San Juan 1:1 upang tukuyin ang Diyos Anak; ganito rin ang kahulugan ng 'salita' (o sa Latin ay verbum) upang tukuyin ang kaalaman, kuru-kuro, at kahulugan.

Ang malay-tao na umuunawa sa kaniyang salita ay hinahangad din ang kaniyang ipinababatid. Ang malay-tao na kayang unawain ang kaniyang persona, at sabay pinagmumunihan ang kaniyang malay-tao, ay hinahangad din sa kaniyang inuunawa. Ito ang kilos ng pag-ibig na mula sa damdamin. Ganito rin ang sinasabi sa Bagong Tipan ukol sa Anak ng Diyos na siya ay pag-ibig (1 Juan 4:8, 16); ngunit kahit hindi pilitin ang teksto, maaari rin itong gamitin upang tukuyin ang Espiritu Santo. Kaya masasabi na sa kilos ng malay-tao (sa kaniyang kakayahan ng unawain ang kaniyang persona at sabay ibigin at hangarin ito), masusumpungan ang isang pagpapahiwatig sa Espiritu Santo.

Ngunit tila may mali sa analogia ni Agustin. Ang nabanggit na kilos ng malay-tao (ang kaniyang pagmumuni-muni sa kaniyang pag-unawa at ang sabay na paghangad rito) ay tila tumutukoy lamang sa banal at hindi sa Ama, Anak, at Espiritu Santo. Ngunit kung babasahin nang maigi si Agustin, mauunawaan na ang salita ng Diyos ay maaaring tumutukoy sa kahit na sinong persona (Ama, Anak, o Espiritu Santo) o sa Banal na Santatlo. Ayon

${ }^{25}$ Tila ganito rin ang sinasabi ni Ferriols. "Ang conceptus ay isang inilihi at binuo sa sinapupunan ng ina." Ferriols, Pambungad sa Metapisika, 3. 


\section{TAO BILANG TAGPUAN}

sa kaniya, ang salitang 'Diyos' ay isa lamang pagpapahiwatig sa 'diwa' ng Diyos; habang ang mga pangalang pantangi naman ay naghahayag ng kanilang ugnayan sa isa't isa. Ang ugnayan ng Banal na Santatlo sa isa't isa ang siyang nagpapatanyag at nagbubukod sa kanila. Kung ganoon, sa kilos ng malay-tao, mahihiwatigan ang ugnayan ng Banal na Santatlo; habang ang malay-tao mismo naman ay maaaring tumukoy sa diwa ng Diyos. Ibig sabihin, ang katagang malay-tao ay hindi tumutukoy sa kahit na anumang ugnayan hanggang hindi ito tahasang ipinahihiwatig.

Upang makahanap ng mas akmang analogia, kinakailangan ni Agustin na baguhin ang katagang pag-unawa at gumamit ng isang kataga na tumutukoy sa kakayahan ng malay-tao na pag-isipan ang kaniyang sariling isip; tinawag niya itong kilos ng malay-tao na gunita. Upang maintindihan ang pagkaangkop na ito, kinakailangang alalahanin na ang pag-unawa (gaya ng pag-alam at pagkilala) ay tumutukoy rin sa paggunita; ang mga katagang Latin para sa isip at unawa (mens) at gunita (memoria) ay tila may pagkakaisa. Samakatuwid, nakumpleto ni Agustin ang analogia ng Banal na Santatlo sa pamamagitan ng tatluhang kilos ng malay-tao: paggunita, pag-unawa, at paghangad sa kaniyang sariling malay-tao.

Ano ang halaga ng paggunita sa usapin ng persona? Kadalasang ginagamit ang gunita upang tukuyin ang mga bagay na lumipas, na nakaraan, o na tapos na; ngunit ang malay-tao ay laging narito sa kasalukuyan ng ating persona. Ngunit para kay Agustin, ang paggunita sa persona ay may pagka-di-karaniwan (kaugnay ng panahon) at sumasaatin ngayon. Sinipi niya si Virgil upang makita ang karanasan ni Ulysses sa kuweba ni Polyphemys. Makikita sa kuwentong ito kung papaano hindi kinalimutan ni Ulysses ang kaniyang persona. Sa ganiyang pamamaraan din natin maaaring gunitain ang ating mga persona. Ito ang tinatawag natin sa wikang Ingles na "presence of mind" o ang paggunita sa persona na tumutukoy rin sa pangunahing presensiya ng isip sa kaniyang sarili. Ang malay-tao ay tumutukoy sa pagkabatid sa persona na masusumpungan lamang sa pamamagitan ng paggunita sa kaniyang persona. ${ }^{26} \mathrm{Ngunit}$ hindi ba ito rin ang kahulugan ng pag-unawa sa persona? Hindi ba parehas lamang ang tinutukoy ng dalawang kilos (paggunita at pag-unawa) na ito ng malay-tao? Upang tugunan ang pagsalangsang na ito, itinuon ni Agustin ang ating pansin sa iba pang kilos ng kaluluwa na tila may santatlong balangkas din. Ito ang mga pandama at ang mga hangarin na tumutuon sa mga nasa labas ng tao. Kasama rin dito ang mga iba't ibang nibel ng pagtingin, at ang paggunita sa mga ordinaryong bagay na atin ng nakita, naaalala, at nadanas.

${ }^{26}$ May malawak na paglalapat sa gunita sa: Agustin, Confessions, trans. by Henry Chadwick (Oxford: Oxford University Press, 2009), Book X, Chapter 17.

(c) 2018 Mark Joseph T. Calano

https://www.kritike.org/journal/issue 22/calano june2018.pdf

ISSN 1908-7330

(cc) BY-NC-ND 
Para kay Agustin, ang malay-tao ay analogia ng Banal na Santatlo hangga't ito ay kamalayan ng sarili nitong presensiya at paggunita; sa pamamagitan ng pagbaling sa sariling presensiya at paggunita, dumarating tayo sa nibel ng sariling pag-unawa. Hindi ba at ganito rin ang ugnayan ng Anak na sumilang (at hindi ginawa) sa Ama bago pa nagkapanahon, Diyos buhat sa Diyos na siya rin, Walang hanggang Salita? Sa pagbaling sa ating malay-tao, nauunawaan natin ang natatanging pagkakaisa at pagkakapantay ng ating sariling paggunita at pag-unawa; gayon din ang kanilang hangganan at uwian sa ating kalooban. Kung mauunawaan natin ang balangkas na ito, masusumpungan din natin ang katotohanan ng banal. Sa balangkas din na ito masusumpungan kung papaanong ang sariling paggunita ng malay-tao ay bumubuo sa kaniyang sariling pag-uunawa at sumasabay rin sa kilos ng kaniyang sariling pag-ibig o paghangad. Ang pagibig at paghangad na ito ang siyang nagdudulot ng pagtanggap at pagpapahalaga sa persona na sabay kapantay at uwian din ng kaniyang persona. Dito nakukumpleto ang tatlong kilos ng malay-tao: pag-unawa, paggunita, at paghangad. Ang huling kilos ng malay-tao ay hindi mapaguusapan gamit ang konsepto o ang analogia ukol sa pagpapaanak o paglikha; sa ganyang pamamaraan din natin hindi maiintindihan ang Espiritu Santo na nanggaling sa-at hindi ipinanganak o nilikha ng-Ama at ng Anak.

May halaga ang sinasabi ni Agustin kung uunawain natin ito sa kaniyang sariling balangkas. Malinaw naman na hindi ito ang huling salita ukol sa ugnayan ng Banal na Santatlo at ng mga kilos ng malay-tao. Sinabi na natin sa itaas na naghanap si Agustin ng sapat na analogia sa kaniyang malaytao upang hiwatigan ang ating personal na paggunita, pag-unawa, at paghangad sa Diyos; naipakita na rin natin ito sa pamamagitan ng angkop na paggunita, pag-unawa, at paghangad ng ating sarili. Masusumpungan ang Diyos sa kilos ng ating malay-tao sa ating personal na kalooban; at matatagpuan natin ang ating personal na kalooban sa pamamagitan ng pakikipagtagpo sa Diyos. Ito rin ang kahulugan ng mga kataga ni Agustin sa Confessiones na intimior intimo meo na ang salin ay maaaring "mas malapit sa akin kaysa sa aking pinakamalalim na persona." 27 Ang kabuoan ng tao at ng kaniyang kapuwa ay matatagpuan lamang sa patuloy na paghigit sa ating mga sarili patungo sa pinakasaligan ng ating buong pagkatao-ang Diyos.

Ano ang ibig sabihin nito? Na kinakailangang gunitain ang tao bilang imago Dei ay hindi lamang isang obhetong kalagayan. Sa ayaw natin o hindi, nakakamit ang magpakatao. At ito ay nakakamtan lamang ng mga kinapal ng Diyos - biyaya at batay pa rin sa Diyos. Dahil diyan, wala tayong masasabi na obhetong kawangis ng Diyos na maaaring hangarin; bagkus, maaari lamang natin unawain ang pagiging kawangis sa loob ng mga kilos ng

${ }^{27}$ Ibid., III, 6.11.

(c) 2018 Mark Joseph T. Calano https://www.kritike.org/journal/issue 22/calano june2018.pdf ISSN 1908-7330 


\section{TAO BILANG TAGPUAN}

pagdikit at pagkapit, paghawak at paninindigan, pag-anib at pagsangayon sa Diyos na siya lamang uwian at huwaran.

Muli, para kay Agustin, at kaiba ng sinasabi ni Tomas de Aquino at ni Aristoteles, ang angkop na daan patungo sa Diyos ay personal at ang kabatiran nito ay isang panloob na kilos at hindi kailanman isang panlabas na pagtanaw sa isang obheto sa mga nilikha. Natatagpuan ang Diyos sa kaibuturan at kaibhan ng bawat tao. Kapag ginagamit ng tao ang kaniyang kakayahan na gunitain, unawain, at hangarin ang Diyos, hindi nito hinahanap ang banal sa labas at hindi ito naghahanap ng isang unibersal na obheto. Ang ating paggunita, pag-unawa, at paghangad sa Diyos ay hindi mula sa ating paghuli at pagdakip, pag-unawa at pag-intindi, pangamba at pagkabahala na mula sa ating labas; Siya ay intimior intimo meo. Sabi nga sa aklat na Ang Mga Gawa, "Sapagkat sa kaniya tayo ay nangabubuhay, at nagsisikilos, at mayroon tayong pagkatao ... Sapagka't tayo nama'y sa kaniyang lahi" (17:28). Ang Diyos ay nananatiling dahilan at uwian ng lahat na patuloy na naghihintay matagpuan upang makita ang ating totoong kalagayan bilang kapuwa-tao.

Ngunit hindi ito automatiko sapagkat binabaluktot at pinipilipit ng ating karupukang-mana ang ating malay-tao. Gustohin man natin o hindi, ang kaganapan ng ating pagkatao ay posible lamang sa Diyos. Samakatuwid, ang kaganapan ng malay-tao ay patungo sa Diyos at may kinalaman sa Kaniya. Ang kasalanan ang nagsisilbing unang yugto sa paglimot sa Diyos; ang kahihinatnan nito ay ang mga susunod na yugto ng kapinsalaan sa ating paggunita, pag-unawa, at paghangad. Binabago ng kasalanan ang kilos ng pag-unawa at paghangad sa Diyos sa simpleng pag-unawa at paghangad sa sannilikha. Dahil sa pagkakasala at pagbaling sa Diyos, hindi tayo nagiging ganap at angkop sa ating pagkatao at sa ating pakikipagtagpo sa banal; ginagawa tayo nito na walang-kaya, mahina, at basag. Ganito na ba tayo ka desperado? Sa aking palagay, ang sagot ay oo.

Ngunit, "nang dumating ang kapanauhan, ay sinugo ng Diyos ang kaniyang Anak, na ipinanganak ng isang babae, na ipinanganak sa ilalim ng kautusan, upang matubos niya ang nangasa ilalim ng kautusan upang matanggap natin ang pagkukupkop sa mga anak" (Galacia 4:4-5). Ang tao ay kinupkop ng Diyos bilang kaniyang mga anak; binago tayo ng biyaya at kaloob ng Diyos sa pamamagitan ni Kristo Hesus - ang Salita ng Diyos na nagkatawang tao. Iginiit ni Agustin na ang obheto ng ating mapagpalayang pananampalataya ay hindi lamang ang Diyos, kung hindi ay ang katawan ng Salitang nagpakatao; ibig sabihin, tinutukoy ni Agustin ang pagsasakatawan ni Hesus bilang dahilan ng ating paglaya sa kasalanan. Sa pamamagitan ng kaniyang katawan, na dumanas ng kamatayan at muling-pagkabuhay, pinalaya tayo ni Kristo sa kasalanan. Habang mahirap unawain ang pagpapakumbaba at pagpapakatao ni Kristo, nananatiling mahalaga ito para 
kay Agustin sapagkat ang pinakasinaunang kasalanan ay nakakagat sa ating pagkamakasarili, o sa kataga ni Ferriols sa ating "ako-ismo" o "aba-ako!" 28 Bilang tugon sa ating pagkakasala, pagkabaluktot, at pagkakabasag, si Kristo ang naging mukha ng kababaang-loob at kaamuhan at sabay ding nagpaanyaya na tanggapin ang ating mga sarili na tao tulad niya.

Ang pagpapanumbalik ng imahe ay nagsisimula sa ating paggaya kay Kristo (na ating huwaran at kaganapan) sa kaniyang paglinang ng kabutihan; para kay Agustin, ang mga kabutihan na tinutukoy ay halimbawa ng pag-uunawa kung saan ang malay-tao ay matuwid at may sapat na paghiwatig sa Diyos lalo na sa kaniyang pagdanas ng mundo. Ang paglinang ng kabutihan ang siyang gumagabay sa isip upang makarating sa karunungan (sapientia) kung saan natin ginugunita, inuunawa, at hinahangad ang Diyos; sa ganitong kalagayan din nararating ang ating personal na kaganapan. Habang ang kaganapang ating tinutukoy ay laging kulang at disapat sa buhay na ito, nararating naman natin ang kaganapan na ito sa ating pakikiisa sa Diyos na isa sa atin.

Tila makikita kay Agustin kung papaanong ang kilos ng malay-tao ay maaaring maging pinto sa ating pakikipagtagpo sa banal. Ganito rin ang sinasabi ni Ferriols kung bakit tao ang tagpuan. Ngunit dahil sa pagkakasala ay nabaling ang ating malay-tao sa maling bagay, at iniwasto ito ni Kristo; habang ang tao ay naghahangad na maging Diyos, si Kristo naman ang Diyos na naghangad na maging tao. Kay Kristo natin mararating ang kaganapan ng ating pagiging nilikha. Mababasa sa sulat ni Pablo sa mga taga Colosas,

Sapagka't sa kaniya nilalang ang lahat na mga bagay, sa sangkalangitan at sa sangkalupaan, na mga bagay na nakikita at ang mga bagay na di nakikita, maging mga luklukan o mga pagsakop o mga pamunuan o mga kapangyarihan; lahat ng mga bagay ay nilalang sa pamamagitan niya at ukol sa kaniya. (1:16)

Mahalaga ang sinasabi ni Pablo upang itulay ang sinasabi ni Agustin ukol kay Kristo at sa susunod na sasabihin ni Ferriols ukol sa puntong Omega.

\section{Isahang may Pagkakaiba}

Sa kaniyang pagtalakay sa kaisipan ni Teilhard de Chardin, nilinaw ni Ferriols na maraming maaaring pagbasa sa puntong Omega. At ang ikatlo sa mga pagbasa na ito, ay ang posibilidad na ang puntong Omega ay hindi

64.

${ }^{28}$ Ferriols, Pambungad sa Metapisika, 223-224 at sa Ferriols, Pilosopiya ng Rehiliyon, 63-

(C) 2018 Mark Joseph T. Calano

https://www.kritike.org/journal/issue 22/calano june2018.pdf

ISSN 1908-7330

(cc) BY-NC-ND 


\section{TAO BILANG TAGPUAN}

isang impersonal na puwersa kung hindi ay si Kristo. Sinabi rin niya na mahihiwatigan daw ito ng taong marunong tumingin ${ }^{29}$ sa pakikisalamuha at pakikipagtalaban kay Kristo ng mga sumasampalataya sa kaniya. Ngunit papaano nakarating si Ferriols sa pagbasa na ito? Papaano at bakit umuuwi sa tao at sa pakikipagkapuwa tao ang tunay na pakikipagtagpo sa banal?

Sinimulan niya sa pagtalakay ng posibleng sinisimbolo ng puno ng buhay sa konteksto ni at para kay de Chardin. ${ }^{30}$ Sa simbolong ito, sa puno ng buhay, mauunawaan ang kilos ng paghahagilap at paglusot sa konteksto ng ebolusyon ng buong sannilikha. Ngunit, sa paglusot ng tao sa buong proseso na ito, nagkakaroon siya ng isang uri ng pagsasaloob ng ebolusyon. ${ }^{31}$ Tila gaya ng kay Agustin, ang kilos na minumungkahi ni de Chardin (ayon kay Ferriols) ay ang pag-unawa sa ebolusyon bilang isang personal na kilos panloob; isang ebolusyon sa larangan ng diwa at sa loob ng malay-tao. Habang hindi sentro ng buong sanlibutan ang tao, may natatangi naman tayong lugar dito sapagkat ang ebolusyon ay nagpapatuloy sa ating pagmamalay sa ating pagkatao. ${ }^{32}$ Sa isang banda, maaari itong maunawaan bilang isang pagtatangi sa kakayahan natin na bigkasin ang ako na iba pa sa iba pang mga ako-muli, ito yung "ako-ismo." Sa isang banda naman, may pagkasalungat ang kilos ng "ako-ismo" sa palagiang pagkukumpol na napagmamasdan din sa puno ng buhay-ang planetisasyon. ${ }^{33}$ Ang "akoismo" kasama ng isang makapunso na pagkukumpol ay lihis na pamamaraan sa loob ng kalawakan-panahon sapagkat binibigyan nito ng diin ang indibiduum at hindi ang persona (na maka-tao at laging sensitibo sa kilos ng malay-tao). ${ }^{34}$ Ano itong persona na ito? Paliwanag ni Ferriols: "ang persona ay hindi isang sentrong nagsasara sa sarili, kundi sentro na, habang lumalalim ang pagsesentro sa sarili, lalong nakikiisa sa mga ibang sentro." 35 Ang sentro na yan na tinutukoy ni Ferriols ay ang puntong Omega.

Tila mahalaga ang papel na ginagampanan ng persona sa patuloy na pakikipag-ugnay sa iba pang mga persona sa kanilang katipunan na tinawag na puntong Omega.

Sa Omega tumutungo ang malay-tao. Doon nakikipagtalaban ang isang sentrong malay-tao sa mga

${ }^{29}$ May mahabang sipi si Ferriols sa Pambungad sa Metapisika mula sa Le phenomene humain ni Teilhard de Chardin ukol sa kahalagahan ng wastong pagtingin. Ibid., 211.

${ }^{30}$ Ferriols, Pambungad sa Metapisika, 214.

${ }^{31}$ Ibid., 217-219,

32 Ibid., 223.

33 Wilhelm P.J. Strebel, “Ang Konsepto ng Planetisasyon ni Teilhard de Chardin: Isang Pagssumubok Bigkasin ang Meron," in Perspectives in the Arts and Humanities Asia (Special Issue: Philosophy and Roque J. Ferriols, SJ), 5:1 (2015), 55-67.

${ }^{34}$ Ferriols, Pambungad sa Metapisika, 225.

${ }^{35}$ Ibid., 225.

(C) 2018 Mark Joseph T. Calano

https://www.kritike.org/journal/issue 22/calano june2018.pdf

ISSN 1908-7330

(c) BY-NC-ND 
ibang sentrong malay-tao, kaya't doon patindi nang patindi ang pagka-persona ng bawat sentro. Ang Omega'y sentro ng personalisasyon. Kaya't hindi isa pang sentrong nakikihalubilo sa ibang mga sentro ang Omega. Hindi rin isang lumitaw mula sa puno ng buhay, hindi produkto ng ebolusyon ang Omega. Ang Omega ay super-sentro: katanyagan, sukdulan ng pagka-persona, ng kasarinlan. ${ }^{36}$

Mahalagang intindihin ang binabanggit ni Ferriols dito kung papaanong ang paglitaw ng malay-tao at ng mga kilos nito ay bahagi ng pagtatalaban ng paghahagilap at paglulusot. Hindi ba ganito ang pagbubuo ng iba't ibang kabutihan at kagalingan (arête) ayon kay Aristoteles? ${ }^{37}$ Sapagkat bahagi tayo ng pandaigdigang kilos ng hagilap-lusot, paliwanag ni Ferriols, taglay raw natin sa ating kalooban ang buong paghahakbang ng sanlibutan. Sa ating pagkatao maaaninaw ang kurba ng paglusot ng persona (at malay-tao) sa patuloy at walang-patid na lugar at punto ng tagpuan (Omega); sa puntong Omega, merong kilos ng pagtipon at pag-iingat sa malay-tao.

Taga-tipon at taga-ingat, iyan ang gawain ng Omega na patuloy na nangangalaga sa ating malay-tao. Ibig sabihin, habang lumalapit tayo sa puntong Omega, lalong naisasakatuparan ang ating kamalayan bilang tao (at kapuwa-tao). Nilinaw ni Ferriols na hindi matutunaw ang sarili sa harap ng isang Dakilang Lahat; hindi tayo nawawala sa pagtitipon ng Omega. Bagkus, palagiang magkasama ang kilos ng pagkakaisa at pagkakaiba sa ating pakikipagtagpo sa puntong Omega. “Ang pagkakaisa ay nagpapaiba. Kaya't malinaw na sa Omega, tuluyan di-tuluyan, ang pagkakaisa ay lalong nagpapaiba." 38 Kaya ang Omega ay kumikilos sa pamamaraang inaakit ang buong sanlibutan tungo sa kaniyang persona; ngunit ang kilos na ito ay hindi upang tuldukan ang bawat nilalang sa kanilang pagmemeron kung hindi ay upang mas lalong buoin ito. Sa pag-aakit ng Omega ay mayroong pagbubuo ng persona (ng pagkatao) sa harap ng nagsisilapitan, nagsisisalubungan, at nagsisitalaban na mga persona (iba pang kapuwa-tao). Magandang paliwanag ni Ferriols:

Ang puntong Omega ay isang sentrong sukdulan ng kasarinlan: Bukod-tanging sentrong sumisinag mula sa puso

36 Ibid., 226.

37 Aristoteles, Nicomachean Ethics, trans. by David Ross (Oxford: Oxford University Press, 2009 (1980), Book II. Roque Ferriols, “Aristoteles: Tapang at Dunong," in Mga Sinaunang Griyego (Quezon City: Office of Research and Publications, Ateneo de Manila University, 1992), 123-129.

${ }^{38}$ Ferriols, Pambungad sa Metapisika, 226. 


\title{
36 TAO BILANG TAGPUAN
}

\begin{abstract}
ng isang katipunan ng mga sentro .... Sa Omega, ang persona ay kumakawala sa bilangguan ng ako-ismo at nagiging tunay na ako. Ang tunay na $A k o$ ay mas tumutubo kung mas naglalaho ang Ako-ismo. ${ }^{39}$
\end{abstract}

Ngunit ang puntong Omega ay hindi maaaring isang impersonal at obhetibong puwersa lamang. May pagkapersonal ang Omega. Kung kaya ay maaaninagan ang bisa ng pagtitipon na ito sa kilos ng pag-ibig na kilos din naman ng damdamin. Ayon kay Ferriols, sa ating pagmamasid sa buong sannilikha ay maaari tayong matauhan na pag-ibig (o sa itaas ay ginamit natin ang salita na paghangad) ang siyang diwa na nagdurugtong sa bawat isa sa atin. Personal ang kilos na ito kung saan mas lalong lumalabas ang ating pagkakaiba (bilang tao) sa ating pagnanais na magkaisa (bilang kapuwa-tao o kapuwa-nilikha). Sa patuloy na pagpapaliwanag ni Ferriols, sinabi niya na nagaganap ang pag-ibig (o paghangad) kapag personal niyang naunawaan na siya ay bahagi ng "kaisahan ng mga nagkakaiba" at "na lalong nagiging iba sabay na lalong nagiging isa." 40 Tinatapos ng mga pag-uunawa na ito ang lahat ng takot sa harap ng di-malamang bukas; sapagkat ang bukas ay may mukha rin, gaya ng ang ngayon ay may mukha rin, at ito ay pag-ibig. Bumubuo at lumilikha ang puntong Omega, hindi ito naninira.

Dito tayo humahantong sa mga maaaring pagbasa sa puntong Omega na ginamit ko upang simulan ang ikatlong bahagi ng papel na ito. Ang Omega, bilang katipunan ng mga nagkakaiba at nagkakaisa, ay kinakailangang: una, "talagang totoo na noon, ngayon, at saka." 41 Kinakailangang higit sa panahon upang makakilos ng lampas pa sa panahon, ngunit ay sabay dapat na nasa panahon upang tipunin ang buong sannilikha. Pangalawa, "matibay na sentro na hindi-maaring-baliktarin." 42 Ginagapos ng lunduyan ang buong sannilikha sa isang pagkakaisa sa harap ng pagkakaiba at binibigkis ang bawat persona upang hindi na mawalay pa. Pangatlo ay "may bukod-tanging kasarinlan." 43 Hindi maaari na tulad rin ng buong sannilikha o katipunan ng buong sannilikha ang Omega. Kinakailangan na may sariling kasarinlan ang Omega na hiwalay sa buong sanlibutan at sa ganyang kalagayan ay hindi ito umaasa sa at nakabatay sa sanlibutan. Pangapat ay "nasa ibayo ng sanlibutan." ${ }^{44}$ Sa ganitong kalagayan din maaaring makita at mauunawaan ang pag-aaruga at pangangalaga ng Omega sa mga persona na hindi nito winawasak sa patuloy nilang pakikipag-salubong-talab

\footnotetext{
${ }^{39}$ Ibid., 227.

${ }^{40} \mathrm{Ibid}$.

${ }^{41}$ Ibid., 228.

${ }_{42}$ Ibid.

${ }^{43}$ Ibid.

${ }^{44} \mathrm{Ibid}$.
}

(c) 2018 Mark Joseph T. Calano

https://www.kritike.org/journal/issue 22/calano june2018.pdf

ISSN 1908-7330

(c) BY-NC-ND 
dito. Sa huli, binanggit ni Ferriols na iisa lamang ang posible at tunay na Omega-si Kristo. ${ }^{45}$

\section{Pagtatapos}

Tinanong natin sa simula ng papel kung papaanong ang tagpuan ng tao at ng mahal-banal ay makikita sa pagsusumikap ng tao na makipagkapuwa-tao. Dinala tayo ng tanong na ito sa pagtalakay ni Ferriols ukol sa damdamin bilang lente ng pag-unawa, ang Diyos bilang personal, at ang tao sa tao. Sa pagtalakay natin sa tao at sa kapuwa-tao, sinubukan natin tignan ang konsepto ng imago Dei. Dinala tayo nito sa pagtalakay ni Agustin sa balangkas ng malay-tao (paggunita, pag-unawa, at paghangad). Ipinakita niya kung papaano ang mga kilos na ito ay humihiwatig din ng Banal na Santatlo, at kung papaano ang mga kilos na ito ang siya ring nagbubuklod sa atin kay Kristo. At habang kumikilos tayo sa kalooban ng ating pagmamalay, ipinakita rin natin ang pagbubuo na dulot ng isang puwersang patuloy na nag-iimbita at nag-aanyaya sa atin-ang puntong Omega. Ibig sabihin, sa patuloy na pagtatalaban ng pagkakaiba at pagkakaisa, nabubuo ang ating malay-tao na kaisa ng iba pang mga malay-tao at ng buong sannilikha; ang puwersang personal na bumubuo ay siya ring gumagalang sa bawat isa sa atin. Dito mahihiwatigan kung bakit nasabi ni Ferriols "na sa pakikipagkapuwa-tao ay tumutubo ang paniniwala sa banal." 46 Nais ko isara ang papel na ito sa pamamagitan ng paghango sa sulat ni Pablo sa mga tagaEfeso. Hangarin ko:

Na si Kristo ay manahan sa inyong mga puso sa pamamagitan ng pananampalataya; upang kung kayo'y mag-ugat at magtumibay sa pagibig. Ay lumakas upang matalastas pati ng lahat ng mga banal kung ano ang luwang at ang haba at ang taas at lalim, at makilala ang pag-ibig ni Kristo na di masayod ng kaalaman, upang kayo'y mangapuspos hanggang sa buong kapuspusan ng Diyos (3:17-19).

Department of Philosophy, Ateneo de Manila University, Philippines

${ }^{45}$ May mahabang sipi si Ferriols sa Pambungad sa Metapisika mula sa Le phenomene humain ni Teilhard de Chardin ukol sa kahalagahan ng wastong pagtingin. Ibid., 211.

${ }^{46}$ Ferriols, Pilosopiya ng Rehiliyon, 16. 


\section{References}

Agustin, Confessions, trans. by Henry Chadwick (Oxford: Oxford University Press, 2009).

Agustin, On the Trinity, ed. by Gareth B. Matthews, trans. by Stephen McKeena (Cambridge: Cambridge University Press, 2002).

Ang Banal na Kasulatan na Kinalalamnan ng Matanda at Bagong Tipan na Isinalin sa Wikang Tagalog (Manila: Philippine Bible Society, 1982).

Aristoteles, Nicomachean Ethics, trans. by David Ross (Oxford: Oxford University Press, 2009 (1980).

de Aquino, Tomas, Summa Theologica [All Complete $\mathcal{E}$ Unabridged 3 Parts, Supplement $\mathcal{E}$ Appendix, and Annotations], translated by Fathers of the English Dominican Province (New York: NY, Benzinger Brother, Inc., 1948).

Cleofas, Jacklyn, "Konsepto at Pag-asa ayon kay Ferriols," in Perspectives in the Arts and Humanities Asia (Special Issue: Philosophy and Roque J. Ferriols, SJ), 5:1 (2015).

Ferriols, Roque, "Aristoteles: Tapang at Dunong," in Mga Sinaunang Griyego (Quezon City: Office of Research and Publications, Ateneo de Manila University, 1992).

"Karanasang Mahal-Banal," in Magpakatao: Ilang Babasahing Pilosopiko (Quezon City: Office of Research and Publications, Ateneo de Manila University, 1999).

Pambungad sa Metapisika (Quezon City: BlueBooks, Ateneo de Manila University Press, 2014). Pilosopiya ng Relihiyon (Quezon City: BlueBooks, Ateneo de Manila University Press, 2014). Sulyap sa Aking Pinanggalingan, ed. by Leovina Ma. Garcia (Quezon City: Ateneo de Manila University Press, 2016).

"Theological Aspects of Cultural Adaptation," in Pagdiriwang sa Meron: A Festival of Thought Celebrating Roque J. Ferriols, S.J., ed. by Nemesio S. Que and Agustin Martin G. Rodriguez (Quezon City: Office of Research and Publications, Ateneo de Manila University, 1997)

Marion, Jean-Luc "On the Foundation of the Distinction between Theology and Philosophy," ed. by Philippe Capelle-Dumont, trans. by John Carlo P. Uy and Eduardo Jose C. Calasanz, in Budhi: A Journal of Ideas and Culture, 13:1-3 (2009).

Strebel, Wilhelm P.J., "Ang Konsepto ng Planetisasyon ni Teilhard de Chardin: Isang Pagssumubok Bigkasin ang Meron," in Perspectives in the Arts and Humanities Asia (Special Issue: Philosophy and Roque J. Ferriols, SJ), 5:1 (2015). 